\title{
Uyarlanabilir Onaylaşım Algoritması Tabanlı Senkronize Duffing Osilatörünün Donanımsal Gerçeklemesi
}

\author{
Gürkan KAVURAN ${ }^{*}$ \\ ${ }^{1}$ Mekatronik Mühendisliği Bölümü, Teknoloji Fakültesi, Fırat Üniversitesi, Elazı̆̆, Türkiye \\ *1 gurkan.kavuran@ozal.edu.tr
}

(Geliş/Received: 27/05/2019;

Kabul/Accepted: 29/09/2019)

Öz: Bu çalışmada iki etmenden oluşan kaos tabanlı ağ yapısının senkronizasyonu için uyarlanabilir onaylaşım algoritması önerilmiştir. Çok çekerli kaotik osilatörlerden biri olan Duffing Osilatörü ile oluşturulan ağ yapısındaki kaotik etmenlerin senkronizasyonu, çizge kuramı teorisinde gradyan düşüm algoritması kullanılarak sağlanmıştır. Sistemin sayısal gerçeklemesi 32-bit ARM tabanlı mikrokontrolör kartı kullanılarak oluşturulmuştur. Önerilen yöntemin geçerliliği ve doğruluğu hem benzetim çalışmaları ile hem de deneysel olarak ispatlanmıştır.

Anahtar kelimeler: Kaos, Duffing Osilatörü, uyarlanabilir onaylaşım algoritması, çok etmenli sistem.

\section{Hardware Implementation of Synchronized Duffing's Oscillator Based on Adaptive Consensus Algorithm}

\begin{abstract}
In this study, an adaptive consensus algorithm is proposed for synchronizing the chaos-based network structure consist of two agent. Synchronization of the chaotic agents in the network structure created by Duffing Oscillator which is one of the multi-scroll chaotic oscillators is provided by using gradient descent algorithm in the graph theory. The digital implementation of the system was generated by using a 32-bit ARM based microcontroller card. The validity and accuracy of the proposed method has been proved by both simulation studies and experimentally.
\end{abstract}

Key words: Chaos, Duffing Oscillator, adaptive consensus algorithm, multi-agent system.

\section{Giriş}

Otonom sistemlerin gelişimiyle birlikte, gezgin robotların birbirleriyle olan iletişimini düzenleyen işbirlikçi kontrol algoritmaları günümüzde önem kazanmıştır. Özellikle sürü robot sistemlerinde, köle robotların efendi robotu takip kabiliyetlerinin artırılıp, koordineli hareketin sağlanması için literatürde birçok çalışmaya rastlanmıştır. Çok etmenli sistemlerde bulunan robot ekipleri, aynı anda birkaç alt görevi yürüterek tek bir robottan daha hızlı ve etkili şekilde görevi gerçekleştirebilmektedir [1]. Bu yapı bazı robotların belirli görevlerde uzman olarak hareket etmesine de olanak sağlamaktadır. Genellikle doğrusal olmayan yapı sergileyen bu sistemlerin senkron hareketleri, çizge kuramı temeline dayanan onaylaşım algoritmaları kullanılarak sağlanmaktadır. Onaylaşım algoritmaları ile ilgili literatürde gezgin robot ağları, otonom hava/deniz/kara araçları, uydu sistemleri gibi birçok çalışma alanına rastlanmıştır [2-7].

Sistemlerin mühendislikteki davranışını analiz etmek için matematiksel modellere duyulan ihtiyaç yadsınamaz bir gerçektir. Genel olarak, deneysel sonuçlar ile elde edilen doğrusal modeller, zengin matematiksel ifadelerden uzaktır. Bu modellerin çoğu yaklaşık değerlerdir ve sistem tanımlamasında kesin doğrulukla çalışmazlar. Süperpozisyon teoremi ve sebep-sonuç ilişkisi, doğrusal sistemlerden doğrusal olmayanlara geçişte kaybolmaya başlar. Literatürde, kaotik sistemler, bilimsel ve endüstriyel alanlarda çalışılmış doğrusal olmayan alanlardan biridir. Çalışmalar kaotik sistemlerin; biyomedikal, haberleşme, elektromanyetik, görüntü işleme, kriptoloji, güç elektroniği, kontrol, fizik, optimizasyon, mekatronik ve yapay sinir aği gibi birçok uygulamada kullanılabileceğini göstermiştir [5-12]. Kaotik sistemlerin ana özellikleri; periyodik olmayan davranış sergilemeleri ve tam olarak başlangıç koşullarına bağlı olmalarıdır. Literatürde çalışılan kaotik sistemlerden biri de Duffing Osilatörüdür. Fiziksel olarak anlaşılması basit ve uygulaması kolay olan bu osilatörün, elektriksel ve mekaniksel olarak benzetimi yapılmıştır. Duffing Osilatörü, sinüsoidal olarak değişen harici bir giriş sinyali uygulanarak kaotik davranış sergilemektedir. İkinci dereceden dinamik bir sistem olan bu osilatörün durum

\footnotetext{
* Sorumlu yazar: gurkan.kavuran@ ozal.edu.tr. Yazarların ORCID Numarası: ${ }^{1}$ 000-0003-2651-5005
} 
değişkenleri arasındaki ilişki, fiziksel olarak basit harmonik hareketten daha karmaşık bir potansiyele sahip olan sönümlü bir yayın hareketini açıklamaktadır.

Zamana bağlı olarak değişen sistemlerde, sisteme etki eden dinamiklerin sadece belirli giriş ve belirli çıkışlar için istenilen sonucu vermesi bazı durumlarda yetersiz kalmaktadır. Örneğin kaymaya bağlı olarak değişen rotor direnci asenkron motorun hız ve tork denetleyicisinde soruna yol açmaktadır. Ya da uygunsuz hava şartlarında çalışan bir servo mekanizmada, başlangıç şartlarına bağlı olarak tasarlanan geleneksel denetleyici yapısı arzu edilen farklı çalışma şartlarına ayak uyduramaz. Bu durumda değişen harici çalışma şartlarına göre sürekli kendini güncelleyen yapılar, gerek akademik gerekse sanayii camiasında ilgi gören konular arasına girmiștir. Adaptif veya uyarlanabilir kontrol mekanizmaları da denilen bu sistemler, özellikle uçuş kontrol sistemlerinde ilk olarak kullanılmış ve başarılı sonuçlar elde edilmiştir. Modele dayalı uyarlanabilir mekanizmaların yanı sıra, bulanık mantık, yapay sinir ağları veya doğrusal olmayan sistem tabanlı denetleyici yapıları bu sistemlere örnek gösterilebilir.

$\mathrm{Bu}$ çalışmada birbirine akuple olan iki Duffing Osilatörün durum değişkenlerinin senkronizasyonu için uyarlanabilir onaylaşım algoritması önerilmiştir. MATLAB ortamında hazırlanan kod rutinleri kullanılarak sistemin benzetimi gerçekleştirilmiştir. Ayrıca donanımsal gerçekleme 32 bitlik Arduino DUE geliştirme kartı üzerinde sağlanmıştır. Çalışmanın organizasyonu şu şekildedir; ikinci bölümde kaotik osilatörün matematiksel model, temel çizge kuramı yapısı ve önerilen uyarlanabilir onaylaşım algoritması hakkında bilgi verilmiştir. Üçüncü bölümde benzetim çalışmaları, dördüncü bölümde ise gerçek zamanlı donanımsal uygulama çalışmaları verilmiştir. Elde edilen sonuçlar ise son bölümde anlatılmıştır.

\section{Teorik Altyapı}

\subsection{Kaotik osilatörün matematiksel modeli}

1918'de G. Duffing tarafindan sunulan Duffing’in osilatörü, genellikle Denklem 1 ile verilen eşitlik ile ifade edilmektedir.

$$
\ddot{x}(t)-x(t)+\alpha \dot{x}(t)+x^{3}(t)=\delta \cos (\omega t)
$$

Denklem 1 ile ifade edilen eşitlik, endüstriyel makinelerin zorlanmış titreşimlerinin kaotik davranışlarını incelemek için kompleks domende şu şekilde yeniden yazılabilir [16].

$\ddot{z}(t)-z(t)+\alpha \dot{z}(t)+\varepsilon z\left|z^{2}(t)\right|=\dot{\gamma} \cos (\omega t)$

Burada $\gamma^{\prime}=\sqrt{2 \gamma} e^{j \pi / 4}, \gamma, \alpha, \omega$ pozitif tanımlı değişkenler ve $z=x+j y$ kompleks bir fonksiyondur. Denklem 2'de $z=x$, $(y=0)$ ve $\varepsilon=1$ alınırsa, Duffing osilatörünün literatürde bilinen tanımı elde edilir. Denklem 2'yi birinci dereceden otonom diferansiyel denklem formunda ifade edersek,

$$
\begin{aligned}
& \dot{x}(t)=y(t) \\
& \dot{y}(t)=x(t)-x^{3}(t)-\alpha y(t)+\delta \cos (\omega t)
\end{aligned}
$$

olur [17].

\section{2. Çizge kuramı temeli}

Birden fazla bağlantı yoluyla birbirleri arasında bilgi aktarımı yapan bir grup etmenden oluşan ağ yapısında, bilgi transferinin yönü de dâhil olmak üzere bağlantıların düzeni bilgi transfer topolojisi olarak bilinir. Bazı etmenler tarafından alınan bilgiler diğerlerinden daha önemli olabileceğinden, bilgi aktarım topolojisini ve bir etmen ağı için her bağlantının ağırlığını tarif etmek gerekmektedir. Ağın özelliklerini matematiksel olarak göstermemizi sağlayan çizge kuramı, düğüm olarak adlandırılan noktalar ve bu noktaları birleştiren hatlardan oluşan ve geometrik bir bilgi vermeyip, sadece düğümler arasındaki ilişkiyi gösteren çizgiler topluluğudur. Beş etmenden oluşan bir ağ yapısı Şekil 1'de verilmiştir. 


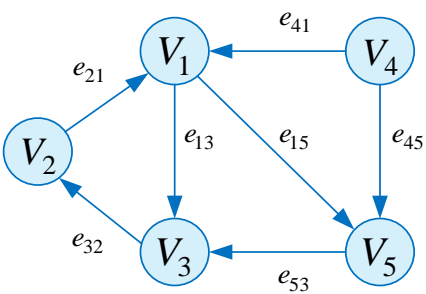

Şekil 1. 5 etmenli yönlendirilmiş çizge yapısı.

Tanım 1. $\boldsymbol{G}(\boldsymbol{V}, \boldsymbol{E})$ çizgesi $n$ etmenden oluşan bir ağı temsil ediyor olsun. A $\breve{g d a}$ bulunan düğümler $\boldsymbol{V}=$ $\left\{\boldsymbol{v}_{1}, \boldsymbol{v}_{2}, \ldots, \boldsymbol{v}_{\boldsymbol{n}}\right\}$ kümesi ve düğümler arası haberleşmeyi tanımlayan kenarlar kümesi ise $\boldsymbol{E} \subseteq \boldsymbol{V} \boldsymbol{x} \boldsymbol{V}$ ile gösterilir. Dügüumleri arasındaki bilgi akışııı ifade eden kenar $\boldsymbol{e}_{\boldsymbol{i j}}=\left(\boldsymbol{v}_{\boldsymbol{j}}, \boldsymbol{v}_{\boldsymbol{i}}\right)$ 'dir ve i düğümünden j düğümüne bir ok ile gösterilir. Her $\boldsymbol{v} \in \boldsymbol{V}$ düğümü için $\boldsymbol{v}$ 'nin derecesi $\boldsymbol{d}(\boldsymbol{v}), \boldsymbol{v}$ düğümünü diğer düğümlerle birleştiren kenarların toplam sayısına eşittir. Bu düğümlerden derecesi en büyük olanı ise, aynı zamanda çizgenin derecesini belirler. Derece matrisi, $\boldsymbol{D}(\boldsymbol{G})=\boldsymbol{d i a g}\left(\boldsymbol{d}_{\mathbf{1}}, \boldsymbol{d}_{2}, \ldots, \boldsymbol{d}_{\boldsymbol{n}}\right)$ olarak ifade edilmektedir [18].

$d(v)=\mid\{u \in V \mid(v, u) \in E$ veya $(u, v) \in E\} \mid$

Tanım 2. Yönlendirilmiş $G(V, E)$ çizgesine ait $n \times m$ boyutundaki insidans matrisi $B(G)=\left[b_{i j}\right], \quad V=$ $\left\{v_{1}, v_{2}, \ldots, v_{n}\right\}$ ve $E=\left\{e_{1}, e_{2}, \ldots, e_{m}\right\}$ için şu şekilde elde edilir.

$b_{i j}=\left\{\begin{array}{cc}+1 & s\left(e_{j}\right)=v_{i} \\ -1 & t\left(e_{j}\right)=v_{i} \\ 0 & \text { diğer durumlar }\end{array}\right.$

Tanım 3. Yönlendirilmiş $G(V, E)$ çizgesine ait $n \times n$ boyutundaki bitişiklik matrisi $A(G)=\left[a_{i j}\right], \quad V=$ $\left\{v_{1}, v_{2}, \ldots, v_{n}\right\}$ ve $E=\left\{e_{1}, e_{2}, \ldots, e_{m}\right\}$ için şu şekilde elde edilir.

$a_{i j}= \begin{cases}1 & \text { eğer }\left(v_{i}, v_{j}\right) \in E \\ 0 & \text { diğer durumlar }\end{cases}$

Önsav 1. Verilen herhangi bir yönlendirilmiş $G(V, E)$ çizgesinin insidans matrisi $B$ ve bitişiklik matrisi $A$ ise, derece matrisi $D$ ile olan ilişki şu şekilde yazılabilir [18].

$B B^{T}=D-A$

Çizge kuramında kullanılan temel iki matristen biri bitişiklik matrisi diğeri ise Laplace matrisidir. Laplace matrisi $L=\left[l_{i j}\right]$ genel olarak şu şekilde ifade edilir.

$\boldsymbol{l}_{i j}=\left\{\begin{array}{cc}\sum_{j=1}^{n} a_{i j} & \text { eğer } i=j \\ -a_{i j} & \text { eğer } i \neq j\end{array}\right.$

Ayrıca Laplace matrisi yönlendirilmiş bir çizge için Denklem 7 ile verilen eşitlik ile de ifade edilebilir. Verilen $G(V, E)$ ağında, etmenlere ait durum değişkenleri kendilerinden ve komşularından aldığ 1 bilgilere göre güncellenir. Literatürde kullanılan sürekli zaman onaylaşım algoritması Denklem 9 ile verilmektedir.

$\dot{x}_{l}(t)=\sum_{j \in N_{i}} a_{i j}(t)\left(x_{j}(t)-x_{i}(t)\right), \quad t \geq 0$

Burada $x_{i}(t) i$. etmenin durum değişkeni, $a_{i j}(t)$ t anındaki bitişiklik matrisinin $(i, j)$. elemanıdır. Denklem 9 matris formunda yeniden düzenlenirse Denklem 10 elde edilir.

$\dot{x}(t)=-L x(t), x(0)=x_{0}$ 


\section{3. Önerilen uyarlanabilir onaylaşım algoritması}

Denklem 3 ile ifade edilen Duffing osilatörü, iki etmenden oluşacak şekilde yeniden düzenlenirse Denklem 11 elde edilir. Sistemi temsil eden ağ yapısı Şekil 2'de verilmiştir. Burada $V_{1}=\dot{x}_{1}(t)$ ve $V_{2}=\dot{x}_{3}(t)$ olarak düşünülebilir.

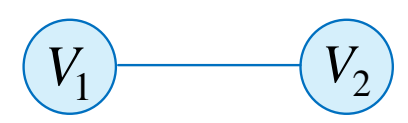

Şekil 2. İki etmenden oluşan ağ yapısı

$$
\begin{aligned}
& \dot{x}_{1}(t)=x_{2}(t) \\
& \dot{x}_{2}(t)=x_{1}(t)-x_{1}^{3}(t)-\alpha x_{2}(t)+\delta \cos (\omega t) \\
& \dot{x}_{3}(t)=x_{4}(t) \\
& \dot{x}_{4}(t)=x_{3}(t)-x_{3}^{3}(t)-\alpha x_{4}(t)+\delta \cos (\omega t)
\end{aligned}
$$

Sistemin durum-uzay formundaki gösterimi Denklem 12'de verilmiştir. Denklem 13'te ise, iki etmenli ağ yapısıyla uyumla olacak şekilde, durum değişkenleri yeniden ifade edilmiştir.

$$
\begin{aligned}
& {\left[\begin{array}{l}
\dot{x}_{1} \\
\dot{x}_{2} \\
\dot{x}_{3} \\
\dot{x}_{4}
\end{array}\right]=\left[\begin{array}{cccc}
0 & 1 & 0 & 0 \\
\left(1-x_{1}^{2}(t)\right) & -\alpha & 0 & 0 \\
0 & 0 & 0 & 1 \\
0 & 0 & \left(1-x_{3}^{2}(t)\right) & -\alpha
\end{array}\right]\left[\begin{array}{l}
x_{1} \\
x_{2} \\
x_{3} \\
x_{4}
\end{array}\right]+\left[\begin{array}{l}
0 \\
\delta \\
0 \\
\delta
\end{array}\right] \cos (\omega t)} \\
& \dot{x}_{1}(t)=\int x_{1}(t)-x_{1}^{3}(t)-\alpha x_{2}(t)+\delta \cos (\omega t) d t \\
& \dot{x}_{3}(t)=\int x_{3}(t)-x_{3}^{3}(t)-\alpha x_{4}(t)+\delta \cos (\omega t) d t
\end{aligned}
$$

İki etmenli yönsüz ağ yapısı için Tanım 1, Tanım 3 'e göre derece ve bitişiklik matrisleri sırasıyla $D=\left[\begin{array}{ll}1 & 0 \\ 0 & 1\end{array}\right]$, $A=\left[\begin{array}{ll}0 & 1 \\ 1 & 0\end{array}\right]$ olarak elde edilir. Önsav 1 ve Denklem 7’ye göre elde edilen Laplace matrisi ise Denklem 14’te verilmiş̧ir.

$L=D-A=\left[\begin{array}{cc}1 & -1 \\ -1 & 1\end{array}\right]$

Burada L matrisi zaman bağlı olarak değişmeyen sabit bir matris olduğundan, sistemdeki ani değişimlere cevabı sağlıklı olmayacaktır. Bu problemin önüne geçmek için, Denklem 10 ile verilen sistem zaman bağglı olarak değişen $k(t)$ uyarlama katsayısı kullanılarak Denklem 15 'deki gibi yeniden yazılabilir.

$\dot{x}(t)=-L k(t) x(t)$

Gradyan düşüm tabanlı senkronizasyon için iki etmen arasındaki farkın karesine bağlı olarak değişen hata fonksiyonu Denklem 16'da verilmiştir [19, 20].

$E(t)=\frac{1}{2} e(t)^{2}$

Burada $e(t)=\dot{x}_{1}(t)-\dot{x}_{3}(t)$ olarak ifade edilmektedir. Hata fonksiyonu $E(t)$ 'yi minimize etmek için $k(t)$ parametresini tahmin eden uyarlama iyileştiricisi ve zamana bağlı olarak değişen uyarlama katsayısı sırasıyla, Denklem 17 ve Denklem 18'deki gibi yazılabilir [21]. 


$$
\begin{aligned}
& \frac{\partial k}{\partial t}=-\sigma \frac{\partial E}{\partial k} \\
& k(t)=-\sigma \int e(t)
\end{aligned}
$$

Denklem 14, 15 ve 18, Denklem 13 içerisinde kullanılarak yeniden yazılırsa, iki etmenli uyarlanabilir onaylaşım tabanlı senkronize Duffing Osilatörünün matematiksel ifadesi Denklem 19'daki gibi olur.

$$
\begin{aligned}
& \dot{x}_{1}(t)=-k(t)\left(x_{1}(t)-x_{3}(t)\right)+\int x_{1}(t)-x_{1}^{3}(t)-\alpha x_{2}(t)+\delta \cos (\omega t) d t \\
& \dot{x}_{3}(t)=-k(t)\left(x_{3}(t)-x_{1}(t)\right)+\int x_{3}(t)-x_{3}^{3}(t)-\alpha x_{4}(t)+\delta \cos (\omega t) d t
\end{aligned}
$$

\section{Benzetim Çalışması}

Bu bölümde önerilen sistemin MATLAB ortamında benzetim modeli oluşturularak, durum değişkenlerinin senkronizasyon problemi incelenmiştir. Kaotik osilatörün modeli, M-file dosyası üzerinden oluşturulan adi diferansiyel denklem takımının çözdürülmesiyle elde edilmiştir. Denklem 18 ile modellenen uyarlama mekanizmasının girişi hata fonksiyonunu oluşturacak durum değişkenleri ve $T_{s}=0.01 \mathrm{sn}$. olarak örneklenmiş zaman fonksiyonu iken çıkışı, uyarlama katsayısı $k(t)$ 'dir. Sistemin MATLAB/Simulink modeli Şekil 3 'te verilmiştir.

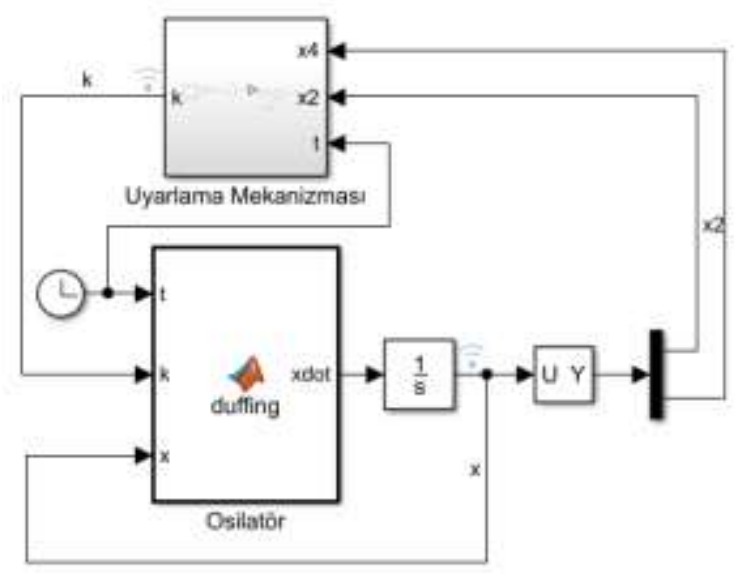

Şekil 3. Önerilen sistemin MATLAB/Simulink blok diyagramı.

Sistem parametreleri $\alpha=0.15, \delta=0.3$ ve $\omega=1$ olan Duffing Osilatörünü ele alalım. Sistem $E_{1}=$ $(1.0728 ; 0), E_{2}=(-0.9061 ; 0)$ ve $E_{3}=(-0.1667 ; 0)$ olmak üzere üç adet denge noktasına sahiptir. Elde edilen özdeğerler sırasıyla; $E_{1}$ için $\lambda_{1,2}=-0.0750 \pm 1.487624 j, E_{2}$ için $\lambda_{1} \approx 1.8547, \lambda_{2} \approx-2.0047$ ve $E_{3}$ için $\lambda_{1} \approx$ $1.1521, \lambda_{2} \approx-1.3021$ olarak elde edilir [16]. $E_{2}$ ve $E_{3}$ denge noktalarının $\lambda_{2}$ ve $\lambda_{3}$ özdeğerleri, kaotik davranış için kararlılık koşulunu sağlayan eyer noktalarıdır. Şekil 4'te Denklem 11 ile verilen Duffing sisteminin $x_{1}(t)$ ve $x_{2}(t)$ düzlemindeki faz yörünge değişimi, başlangıç koşulları $x_{1}(0)=0.21$ ve $x_{2}(0)=-0.7$ için verilmiştir. Şekil 5 'te ise $x_{3}(t)$ ve $x_{4}(t)$ düzlemindeki faz yörünge değişimi başlangıç koşulları $x_{3}(0)=0.31$ ve $x_{4}(0)=0.8$ için gösterilmiştir. Simülasyon süresi $T_{\text {sim }}=250 \mathrm{sn}$. ve örnekleme periyodu ise $h=0.001$ sn.'dir.

Denklem 19 ile verilen sistem için $x_{1}(t)$ ve $x_{3}(t)$ durum değişkenlerinin zamana bağlı olarak kaotik değişimleri Şekil 6'da gösterilmiştir. $T_{\text {sim }}=100$. sn'de devreye alınan uyarlama mekanizması sayesinde iki sistemin yaklaşık olarak $12 \mathrm{sn}$. sonra senkronizasyonu sağlanmıştır. Uyarlama katsayısı bu örnek için $\sigma=0.2$ olarak seçilmiştir. Bu katsayısının büyük seçilmesi senkronizasyon hızını doğru orantılı olarak etkilemektedir. Ancak yüksek değerler, sistemi kararsızlığa sürüklemektedir. Senkronizasyonun ayrıntılı grafiği Şekil 7 'de verilmiştir. Şekil 8'de ise aynı sistem için $x_{2}(t)$ ve $x_{4}(t)$ durum değişkenlerinin zamana bağlı olarak kaotik değişimleri gösterilmiştir. Hata fonksiyonu $e(t)$ ve uyarlama katsayısı $k(t)$ 'nin zamana göre değişimleri Şekil 10 'da verilmiştir. Önerilen yapı sisteme dahil edildikten sonra, hata fonksiyonun giderek azalarak sıfırlandığı, uyarlama 
katsayısının ise sonlu bir süre sonunda yaklaşık olarak 0.5 değerinde sabit kaldığı görülmektedir. Bu değerin sabit kalarak sistemin senkronizasyonunun devam etmesi, önerilen sistemin kararlı olduğunu göstermektedir.

$\mathrm{Bu}$ bölümde tasarımı yapılan ve benzetim sonuçları verilen senkronize kaotik sistemin gerçek zamanlı uygulaması, Bölüm 4’te ayrıntılı şekilde anlatılacaktır.

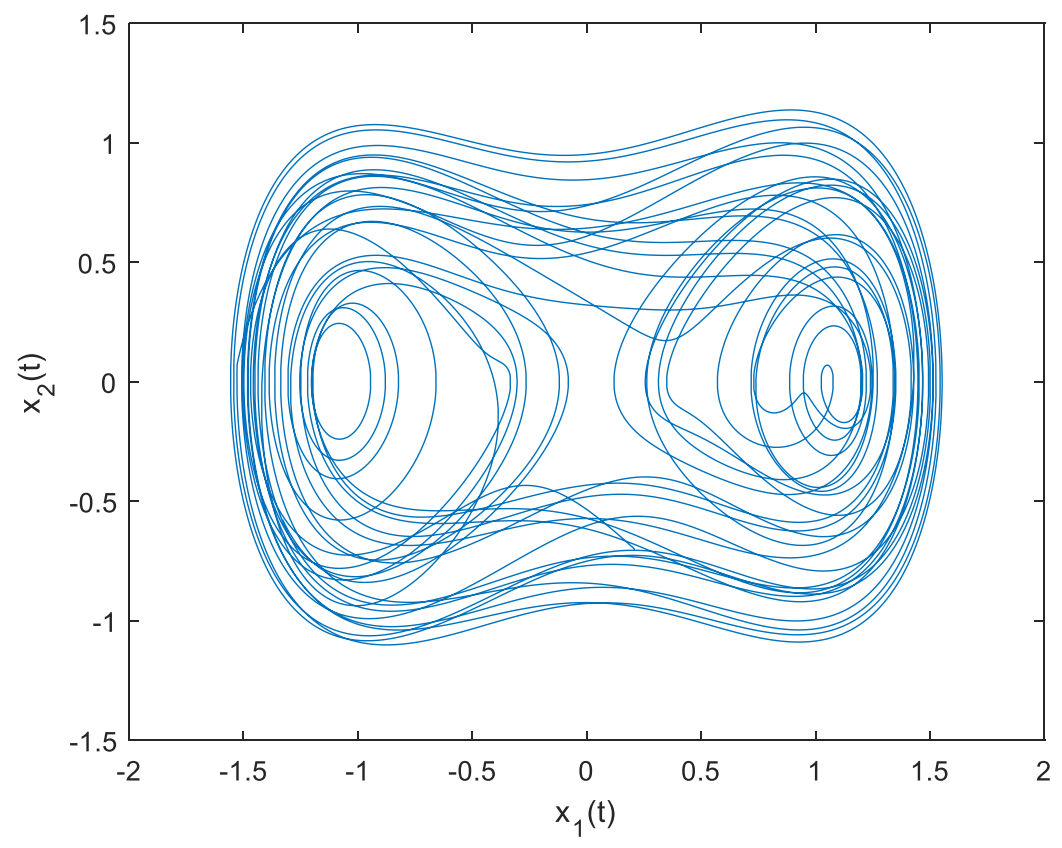

Şekil 4. Denklem 11 ile verilen Duffing sisteminin $x_{1}(t)$ ve $x_{2}(t)$ düzlemindeki faz yörünge değişimi (Sistem parametreleri $\alpha=0.15, \delta=0.3, \omega=1$ ve başlangıç koşulları $x_{1}(0)=0.21$ ve $x_{2}(0)=-0.7$ )

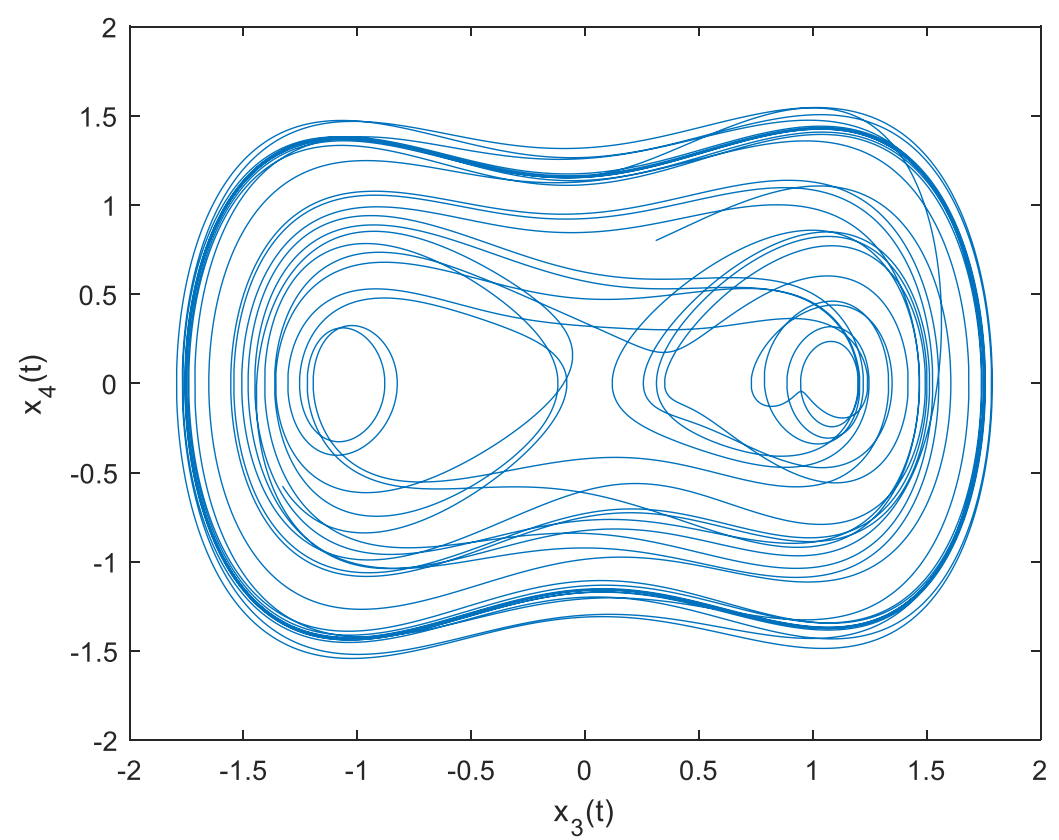

Şekil 5. Denklem 11 ile verilen Duffing sisteminin $x_{3}(t)$ ve $x_{4}(t)$ düzlemindeki faz yörünge değişimi (Sistem parametreleri $\alpha=0.15, \delta=0.3, \omega=1$ ve başlangiç koşulları $x_{3}(0)=0.31$ ve $x_{4}(0)=0.8$ ) 


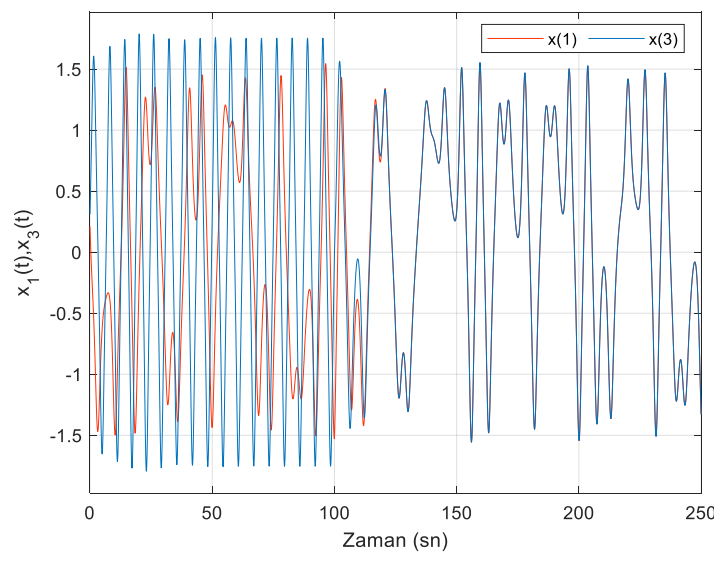

Şekil 6. Denklem 19 ile verilen sistem için $x_{1}(t)$ ve $x_{3}(t)$ durum değişkenlerinin zamana bağl1 olarak kaotik değişimleri $(\sigma=0.2)$.

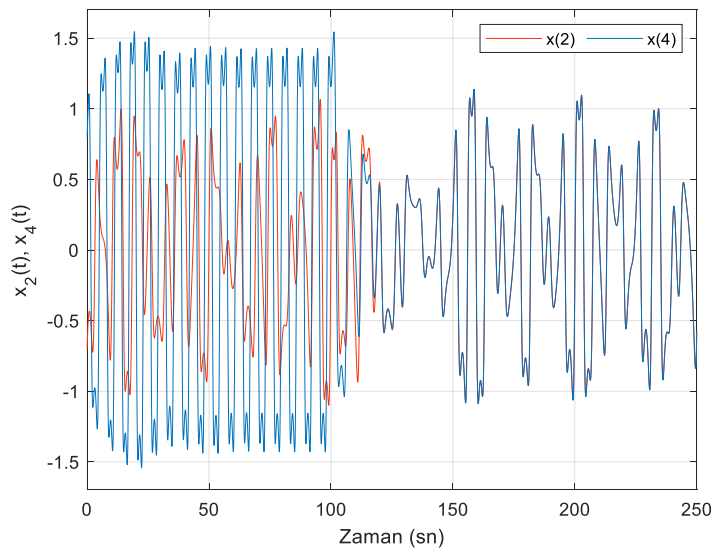

Şekil 8. Denklem 19 ile verilen sistem için $x_{2}(t)$ ve $x_{4}(t)$ durum değişkenlerinin zamana bağl1 olarak kaotik değişimleri $(\sigma=0.2)$.

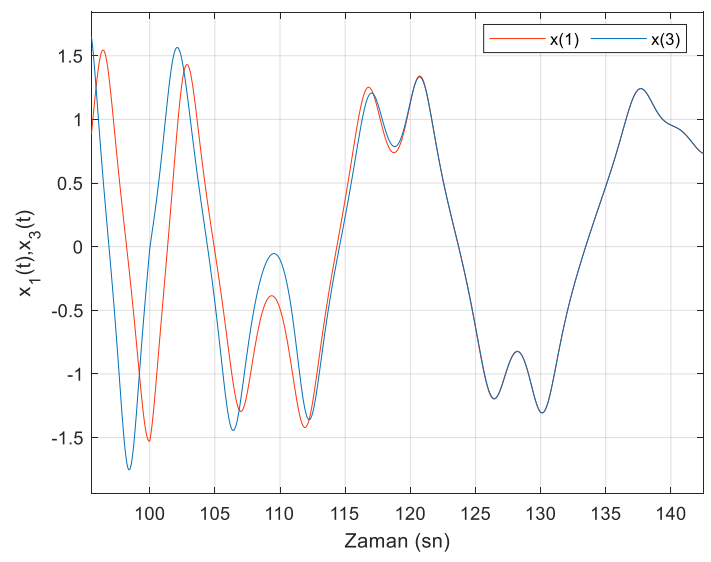

Şekil 7. Şekil 6 ile verilen değişimlerin ayrıntılı gösterimi.

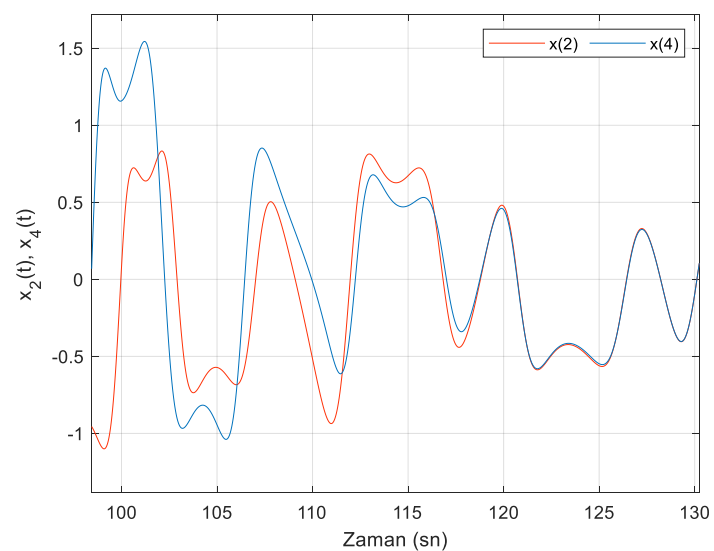

Şekil 9. Şekil 8 ile verilen değișimlerin ayrıntılı gösterimi.

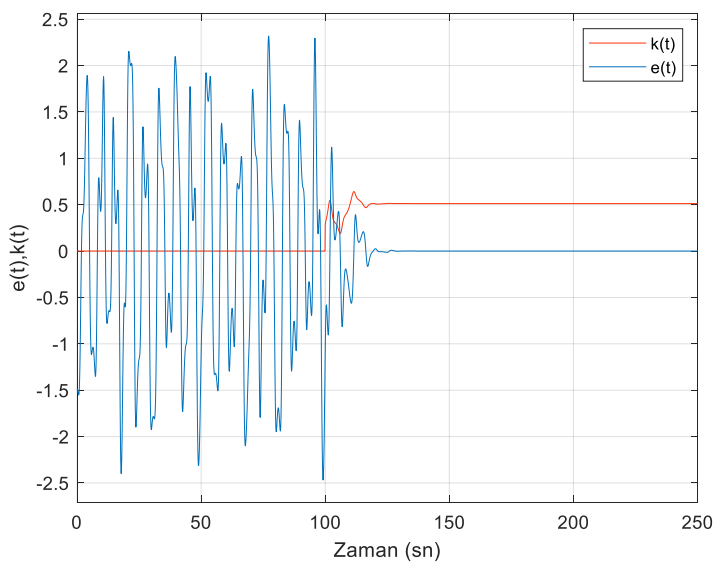

Şekil 10. Hata fonksiyonu $e(t)$ ve uyarlama katsayısı $k(t)$ 'nin zamana göre değişimleri ( $\sigma=0.2$ ). 


\section{Donanımsal Gerçekleme ve Deneysel Sonuçlar}

Sistemin dijital olarak gerçeklemesi için, sayısal yöntemlerden faydalanmak gerekir. Başlangıç değer problemine sahip, adi diferansiyel denklemlerin çözümleri genel olarak Euler, Taylor ve Runge-Kutta metotları kullanılarak elde edilmektedir [22]. Bu çalışmada sistemin çözümü için kullanılan sayısal türev, ileri fark yaklaşım tabanlı Euler yöntemidir. Buna göre Denklem 11 ve 16 kullanılarak elde edilen sistem takımı aşağıda verilmiştir.

$$
\begin{aligned}
& x_{1}\left(t_{i+1}\right)=x_{1}\left(t_{i}\right)+h *\left(x_{2}\left(t_{i}\right)-k\left(t_{i}\right)\left(x_{1}\left(t_{i}\right)-x_{3}\left(t_{i}\right)\right)\right) \\
& x_{2}\left(t_{i+1}\right)=x_{2}\left(t_{i}\right)+h *\left(x_{1}\left(t_{i+1}\right)-\alpha x_{2}\left(t_{i}\right)-x_{1}^{3}\left(t_{i}\right)+\delta \cos (\omega * h * i)\right) \\
& x_{3}\left(t_{i+1}\right)=x_{3}\left(t_{i}\right)+h *\left(x_{4}\left(t_{i}\right)-k\left(t_{i}\right)\left(x_{3}\left(t_{i}\right)-x_{1}\left(t_{i}\right)\right)\right) \\
& x_{4}\left(t_{i+1}\right)=x_{4}\left(t_{i}\right)+h *\left(x_{3}\left(t_{i+1}\right)-\alpha x_{4}\left(t_{i}\right)-x_{3}^{3}\left(t_{i}\right)+\delta \cos (\omega * h * i)\right)
\end{aligned}
$$

Denklem 21 ile verilen türevsel sistem takımının gerçek zamanlı çözümü, Atmel SAM3X8E ARM CortexM3 CPU'yu temel alan Arduino Due mikrodenetleyici kartı üzerinde sağlanmıştır. 32-bit ARM çekirdekli mikrodenetleyici yapısına dayanan bu kart, 54 dijital giriş / çıkış (12'si PWM çıkışı), 12 analog giriş, 4 UART, 84 MHz saat frekansı, USB OTG özellikli bağlantı, 2 DAC (dijital - analog) çıkış özelliğine sahiptir [23]. Tasarlanan sistemin çıkışları DAC arabirimi kullanılarak analog veriye çevrilmiştir. Ölçümler USB arabirimi ile çalışan Analog Discovery 2 Osiloskop kartı kullanılarak PC ortamına alınmıştır. Sistemin genel görüntüsü Şekil 11 'de verilmiştir [24].

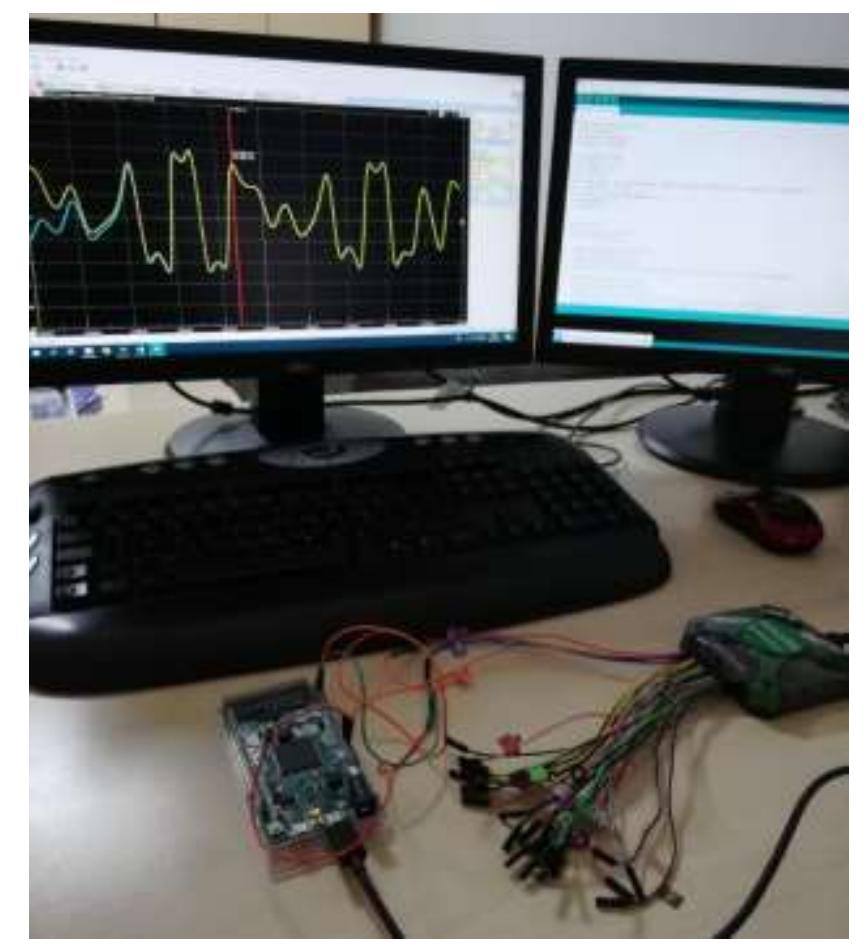

Şekil 11. Donanımsal gerçekleme ve test düzeneği.

Şekil 12'de Denklem 11 ile verilen sistem için $x_{1}(t)$ ve $x_{2}(t)$ durum değişkenlerinin gerçek zamanlı kaotik değişimleri verilmiştir. Başlangıç koşulları $x_{1}(0)=0.21$ ve $x_{2}(0)=-0.7$ ve örnekleme periyodu $h=0.1$ sn.'dir. Sistemin $x_{1}(t)$ ve $x_{2}(t)$ düzlemindeki gerçek zamanlı faz yörünge değişimi ise Şekil 13'te gösterilmiştir. Şekil 14 'te Denklem 19 ile verilen sistem için $x_{2}(t)$ ve $x_{4}(t)$ durum değişkenlerinin gerçek zamanlı kaotik değişimleri verilmiştir. Önerilen uyarlama mekanizması sayesinde iki sistemin yaklaşı k olarak 5.49 sn. sonra senkronizasyonu sağlanmıştır. Uyarlama katsayısı bu örnek için $\sigma=1 \times 10^{-6}$ olarak seçilmiştir. Aynı $\sigma$ değeri için hata fonksiyonu $e(t)$ ve uyarlama katsayısı $k(t)$ 'nin gerçek zamanlı değişimleri Şekil 15'te verilmiştir. Hata fonksiyonunun 
azalarak sıfırlandığı, uyarlama katsayısının ise yaklaşık olarak 0.2 değerinde sabit kaldığı görülmektedir. Farklı $\sigma$ katsayısının senkronizasyon süresine olan etkisini incelemek amacıyla $\sigma=1 \times 10^{-5}$ seçilip durum değişkenlerinin değişimleri tekrar incelenmiştir. Şekil 16'daki $x_{2}(t)$ ve $x_{4}(t)$ durum değişkenlerinin gerçek zamanlı kaotik değişimleri incelendiğinde, bu katsayının 1/10 oranda büyütülmesiyle senkronizasyon süresinin 5.49 sn.'den 1.98 sn.'ye düştüğü gözlemlenmiştir.

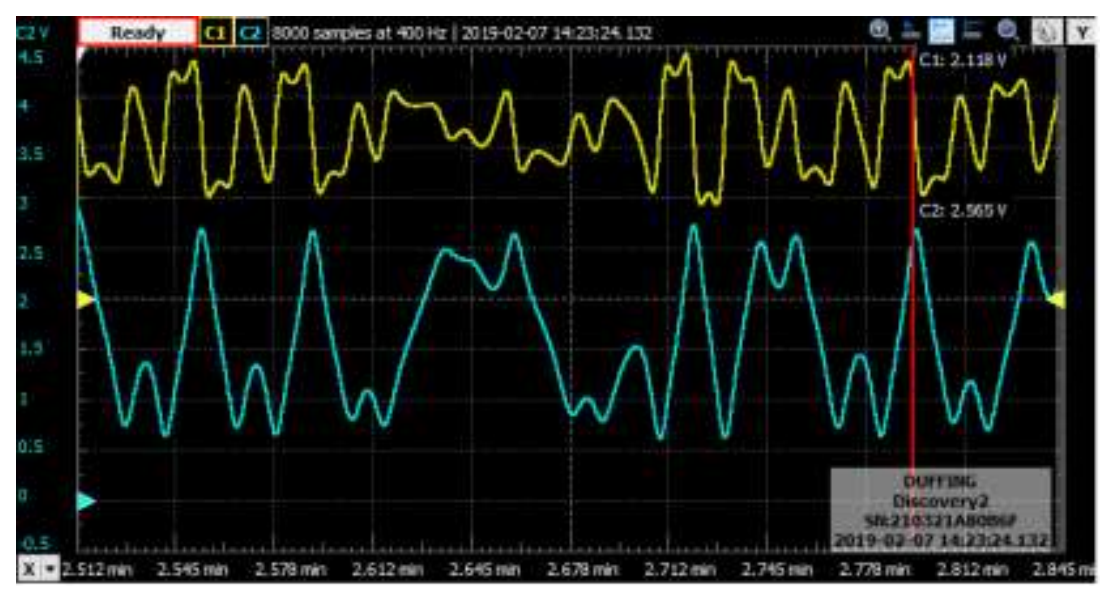

Şekil 12. Denklem 11 ile verilen sistem için $x_{1}(t)$ ve $x_{2}(t)$ durum değişkenlerinin gerçek zamanlı kaotik değişimleri.

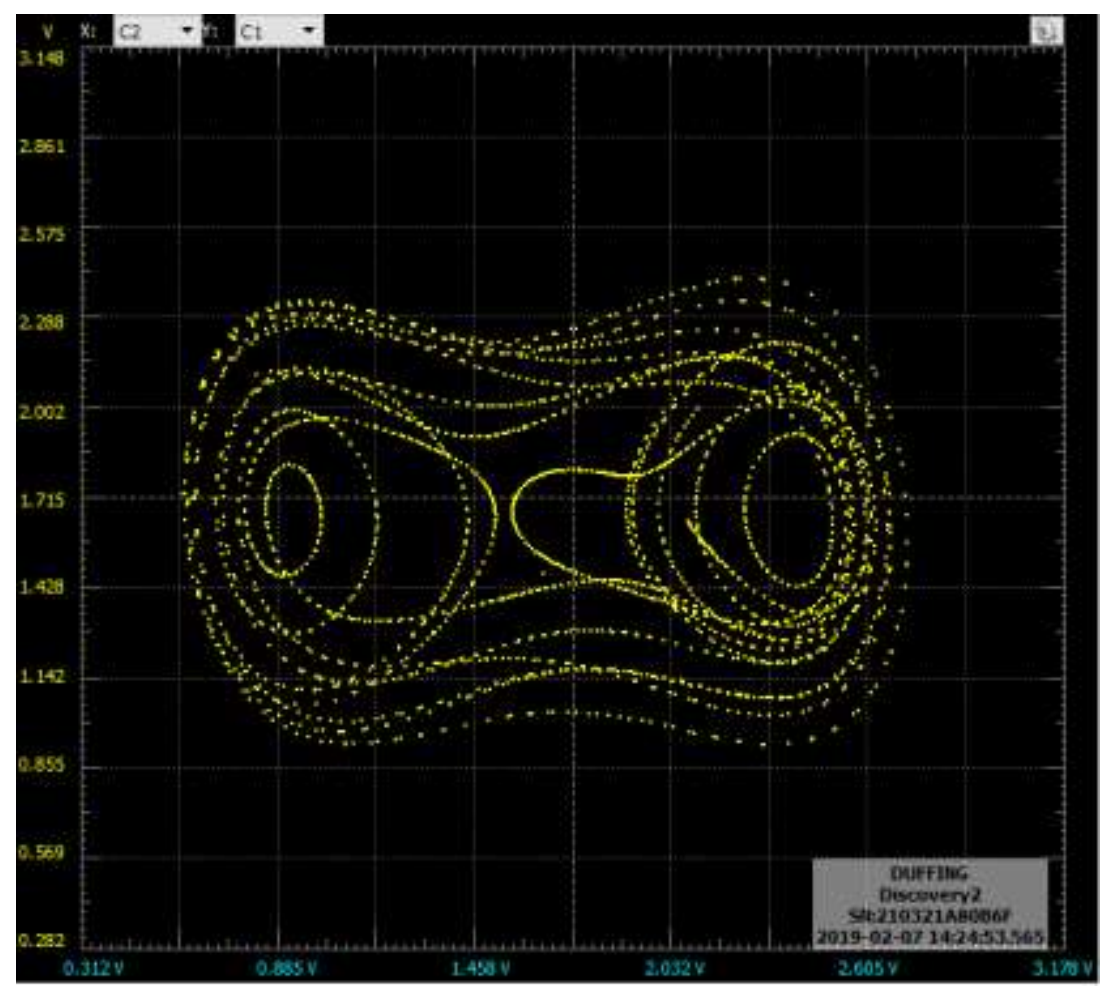

Şekil 13. Denklem 11 ile verilen Duffing sisteminin $x_{1}(t)$ ve $x_{2}(t)$ düzlemindeki gerçek zamanlı faz yörünge değişimi (Sistem parametreleri $\alpha=0.15, \delta=0.3, \omega=1$ ve başlangıç koşulları $x_{1}(0)=0.21$ ve $x_{2}(0)=-0.7$ ) 


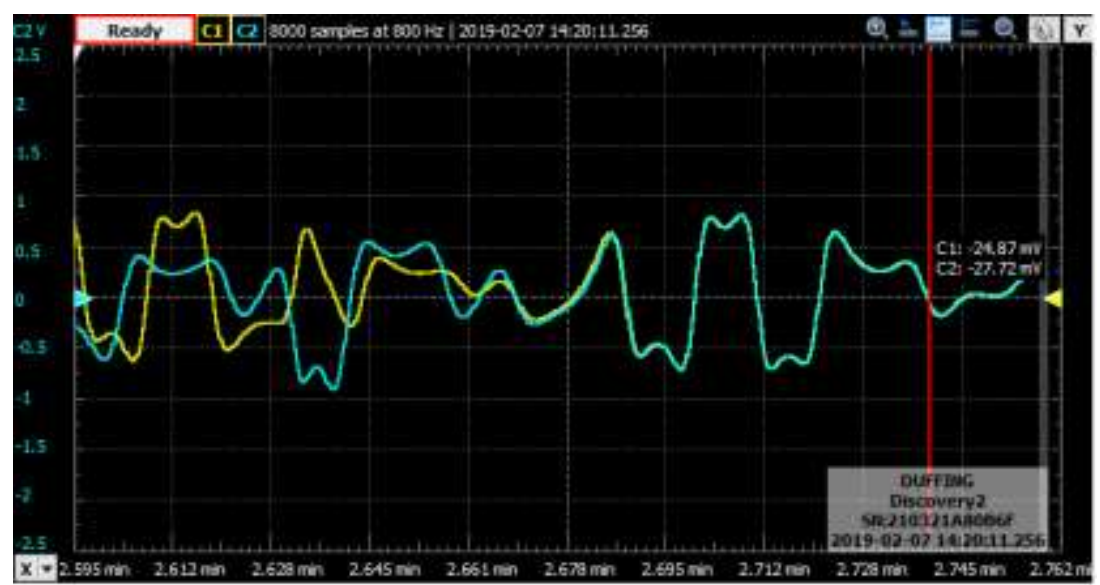

Şekil 14. Denklem 19 ile verilen sistem için $x_{2}(t)$ ve $x_{4}(t)$ durum değişkenlerinin gerçek zamanlı kaotik değişimleri $\left(\sigma=1 \times 10^{-6}\right)$.

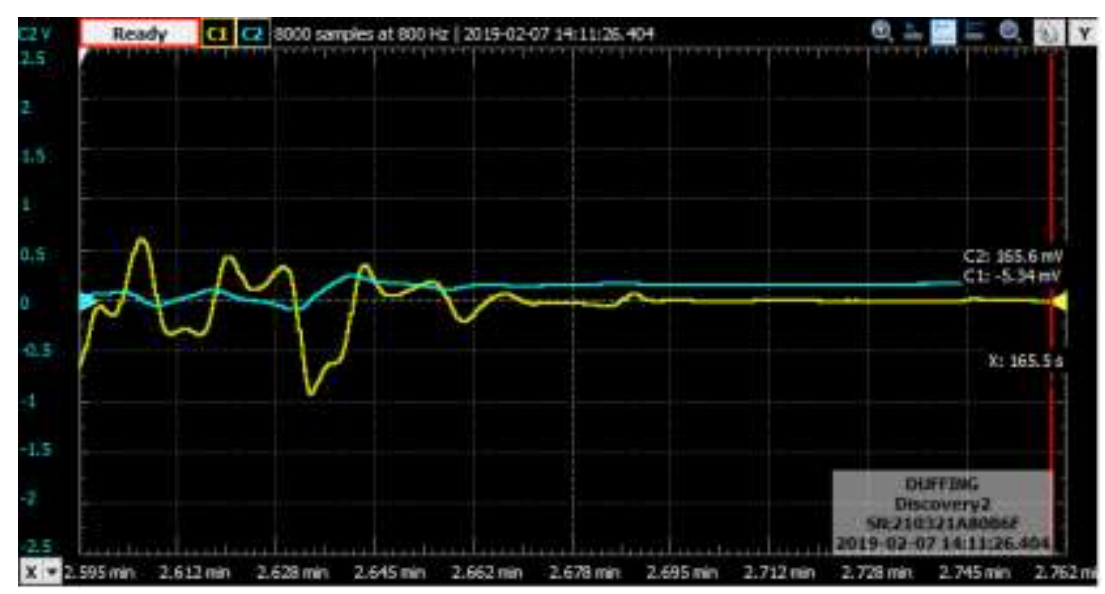

Şekil 15. Hata fonksiyonu $e(t)$ ve uyarlama katsayısı $k(t)$ 'nin gerçek zamanlı değişimleri $\left(\sigma=1 \times 10^{-6}\right)$.

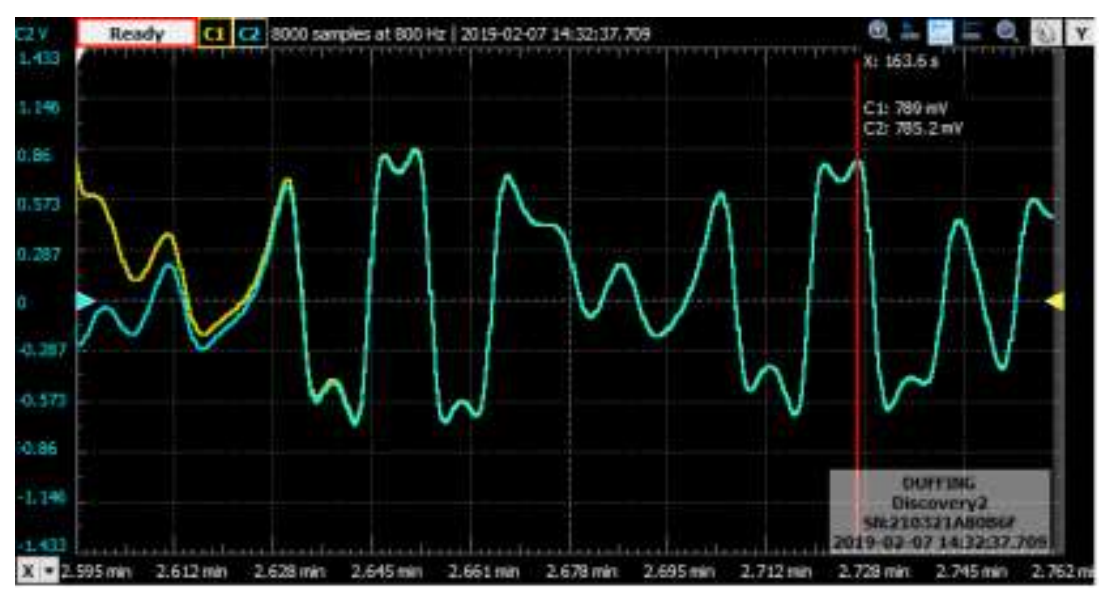

Şekil 16. Denklem 19 ile verilen sistem için $x_{2}(t)$ ve $x_{4}(t)$ durum değişkenlerinin gerçek zamanlı kaotik değişimleri $\left(\sigma=1 \times 10^{-5}\right)$. 


\section{Sonuçlar}

Bu çalışmada birbirine akuple olan iki Duffing Osilatörün durum değişkenlerinin senkronizasyonu için uyarlanabilir onaylaşım algoritması önerilmiştir. MATLAB ortamında hazırlanan kod rutinleri kullanılarak sistemin benzetimi gerçekleştirilmiştir. Ayrıca donanımsal gerçekleme 32 bitlik Arduino DUE geliştirme kartı üzerinde sağlanmıştır. Önerilen algoritmanın gerek benzetim gerekse gerçek zamanlı çalışmada, iki kaotik sistemi senkronize ettiği gözlemlenmiştir. Onaylaşım algoritmalarının gezgin robot ağları, otonom hava/deniz/kara araçları, uydu sistemleri gibi uygulama alanına sahip olduğu göz önünde bulundurulursa, gelecek çalışmalarda sürü hareket kabiliyetine sahip gezgin robot ağlarında bu algoritmanın kullanılması hedeflenmektedir. Ayrıca şifreleme algoritmalarında kullanılan rastgele sayı üreteçlerindeki kaotik altyapıya da katkı sağlayacağı düşünülmektedir.

\section{Kaynaklar}

[1] Jadbabaie A, Lin J, Morse AS. Coordination of groups of mobile autonomous agents using nearest neighbor rules. Proceedings of the 41st IEEE Conference on Decision and Control; 2003; 2953-2958.

[2] Zhang Y, Li S. Distributed Biased Min-Consensus with Applications to Shortest Path Planning. IEEE Trans. Automat. Contr. 2017; 62(10): 5429-5436.

[3] Schwager M, Rus D, Slotine J-J. Decentralized, Adaptive Coverage Control for Networked Robots. Int. J. Rob. Res. 2009; 28(3): 357-375.

[4] Nguyen KD, Dankowicz H. Synchronization and consensus of a robot network on an underactuated dynamic platform. 2014 IEEE/RSJ International Conference on Intelligent Robots and Systems; September 2014; 117-122.

[5] Dong X, Yu B, Shi Z, Zhong Y. Time-Varying Formation Control for Unmanned Aerial Vehicles: Theories and Applications. IEEE Trans. Control Syst. Technol. 2015; 23(1): 340-348.

[6] Zhongkui Li, Zhisheng Duan, Guanrong Chen, Lin Huang. Consensus of Multiagent Systems and Synchronization of Complex Networks: A Unified Viewpoint. IEEE Trans. Circuits Syst. I Regul. Pap. 2010; 57(1): 213-224.

[7] Erkan ÖF, Akar M. Continuous-time analysis of multi-agent systems with multiple consensus equilibria. Pamukkale Univ. J. Eng. Sci. 2016; 22(8): 666-670.

[8] Hernán Díaz M, Maureira F, Flores E, Córdova F. Intra and inter-hemispheric correlations of the order/chaos fluctuation in the brain activity during a motor imagination task. Procedia Computer Science; Jan 1, 2018; 456-463.

[9] Wang L, Long X, Aarts RM, van Dijk JP, Arends JBAM. A broadband method of quantifying phase synchronization for discriminating seizure EEG signals. Biomed. Signal Process. Control 2018; .

[10] Pandey A, Singh B, Saini BS, Sood N. A novel fused coupled chaotic map based confidential data embedding-thenencryption of electrocardiogram signal. Biocybern. Biomed. Eng. 2019; 39(2): 282-300.

[11] Sahin ME, Cam Taskiran ZG, Guler H, Hamamci SE. Simulation and implementation of memristive chaotic system and its application for communication systems. Sensors Actuators, A Phys. 2019; 290(1): 107-118.

[12] Anbing Zhang, Yichun Xie. Chaos Theory-Based Data-Mining Technique for Image Endmember Extraction: Laypunov Index and Correlation Dimension (L and D). IEEE Trans. Geosci. Remote Sens. 2014; 52(4): 1935-1947.

[13] Özkaynak F, Özer AB. Cryptanalysis of a new image encryption algorithm based on chaos. Optik (Stuttg). 2016; 127(13): 5190-5192.

[14] Chen L, Ma B, Zhao X, Wang S. Differential cryptanalysis of a novel image encryption algorithm based on chaos and Line map. Nonlinear Dyn. 2017; 87(3): 1797-1807.

[15] Alzahrani A, Shamsi P, Ferdowsi M, Dagli CH. Chaotic Behavior in High-Gain Interleaved DC-DC Converters. Procedia Computer Science; January 1, 2017; 408-416.

[16] Petras I. Fractional-Order Nonlinear Systems: Modeling, Analysis and Simulation. Springer Press, 2011.

[17] Gao X, Yu J. Chaos in the fractional order periodically forced complex Duffing's oscillators. Chaos, Solitons and Fractals 2005; 24(4): 1097-1104.

[18] Gallier JH. Spectral Theory of Unsigned and Signed Graphs Applications to Graph Clustering: a Survey. , 2018.

[19] Kavuran G, Ates A, Alagoz BB, Yeroglu C. An experimental study on model reference adaptive control of TRMS by error-modified fractional order MIT rule. Control Eng. Appl. Informatics 2017; 19(4): 101-111.

[20] Alagoz BB, Kavuran G, Ates A, Yeroglu C. Reference-shaping adaptive control by using gradient descent optimizers. PLoS One 2017; 12(11): e0188527.

[21] Alagoz BB, Tepljakov A, Kavuran G, Alisoy H. Adaptive Control of Nonlinear TRMS Model by Using Gradient Descent Optimizers. 2018 International Conference on Artificial Intelligence and Data Processing (IDAP); 2018;1-6.

[22] Kincaid D, Cheney W. Numerical Analysis:Mathematics of Scientific Computing., 3rd Revise. American Mathematical Society, 2002.

[23] Arduino. Arduino DUE Teknik Özellikler.[Online]. Available: https://store.arduino.cc/usa/due. [Accessed: 23-May2019].

[24] Digilent. Analog Discovery 2 Teknik Özellikler. . [Online]. Available: https://store.digilentinc.com/analog-discovery2-100msps-usb-oscilloscope-logic-analyzer-and-variable-power-supply/. [Accessed: 23-May-2019]. 